\title{
Anorexia due to Depression in the Elderly From the Viewpoint of Primary Care
}

\author{
Syuichi Tetsuka ${ }^{\mathrm{a}, \mathrm{b}}$, Mieko Otsuka ${ }^{\mathrm{a}}$, Ritsuo Hashimoto ${ }^{\mathrm{a}}$, \\ Hiroyuki Kato ${ }^{\text {a }}$
}

\begin{abstract}
Japan is currently an aging society with a huge proportion of elderly citizens. Consequently, the incidence and severity of anorexia and depression are predicted to increase in the future. In fact, at present, anorexia is common in the elderly. Anorexia is one of the major symptoms of depression in the elderly. More than $90 \%$ of patients with depression are thought to visit primary care physicians and the departments such as general medicine first instead of psychiatrists. Therefore, primary care physicians need to be vigilant for signs of depressive disorder to prevent suicide. Elderly patients have few negative emotions, such as feeling depressed, sad, or any other subjective drops in mood; this implies that elderly individuals might find it difficult to explain their symptoms to others and that the people around them might hardly notice any symptoms of depression. An 84-year-old woman started to gradually lose appetite. She has undergone various examinations, but an obvious physical organic abnormality was not found. Upon admission to our hospital, her weight was $38 \mathrm{~kg}$ and body mass index was $16.9 \mathrm{~kg} / \mathrm{m}^{2}$. Based on diagnostic criteria and further evaluation, we diagnosed her as anorexia due to depression. After biopsychosocial approach including appropriate social support, as well as medical and mental therapies, her appetite improved gradually and she was able to regain her weight eventually. Elderly individuals have a tendency to get depression due to biologic and psychosocial causes. Many patients with depression are thought to visit primary care physicians and the departments such as general medicine first instead of psychiatrists. Therefore, the efficiency of primary care is underscored and primary care physicians need to be vigilant in detecting depression and preventing suicide. We think that general medicine will have a more important part in medical practice in the future in Japan.
\end{abstract}

Keywords: Anorexia; Depression; Elderly; Primary care; Biopsychosocial; General medicine

Manuscript accepted for publication March 17, 2017

aDepartment of Neurology, Hospital of International University of Health and Welfare, 537-3, Iguchi, Nasushiobara, Tochigi 329-2763, Japan

${ }^{b}$ Corresponding Author: Syuichi Tetsuka, Department of Neurology, Hospital of International University of Health and Welfare, 537-3, Iguchi, Nasushiobara, Tochigi 329-2763, Japan. Email: syuichi@jichi.ac.jp

doi: https://doi.org/10.14740/jmc2794w

\section{Introduction}

The lifetime prevalence of depression is estimated to be 1 in 10 men and 1 in five women, implying that it is not a rare condition. More than $90 \%$ of patients with depression are thought to visit primary care physicians and the departments such as general medicine first instead of psychiatrists. Therefore, primary care physicians need to be vigilant for signs of depressive disorder to prevent suicide. Elderly individuals may be easy to fall into a depressed state because of biological changes in the brain and body aging; experiences of loss of a spouse, friend, etc.; and need for stable biopsychosocial status in health and financial aspects. In fact, the rate of suicide is higher in older age groups than in the other generations. The incidence of dementia also increases with aging. Although depression and dementia are common in the elderly age group, recognition and diagnosis of such diseases in the clinical setting might be difficult.

Eating disorders occur most commonly in adolescents and are rarely newly diagnosed after the age of 40 years [1]. However, anorexia is also one of the major symptoms of depression in the elderly. In addition to an impact on quality of life, significant morbidity and mortality are associated with anorexia. Individuals diagnosed with an eating disorder may suffer from osteoporosis, electrolyte imbalance, altered gastrointestinal motility, hormonal shifts, leukopenia, seizures, and cardiac arrhythmia. Japan is currently an aging society with a huge proportion of elderly citizens. Consequently, the incidence and severity of anorexia and depression are predicted to increase in the future. In fact, at present, anorexia is common in the elderly. The social burdens, financial strain, and the high level of physical and cognitive intactness required for meal preparation all contribute to decreased appetite and food intake in older people [2]. Anorexia can also be a symptom of physical illness or above-mentioned depression $[3,4]$. Anorexia tardive, a related disorder specific to the elderly, is marked by loss of appetite and significant weight loss, but has more depressive symptoms, less disturbed body image, and preoccupation with eating behavior compared with anorexia nervosa. This condition may be secondary to complicated bereavement as a variant of a geriatric depressive episode [5].

In this report, we present a case of an elderly woman who visited a primary care physician first and was eventually diag- 

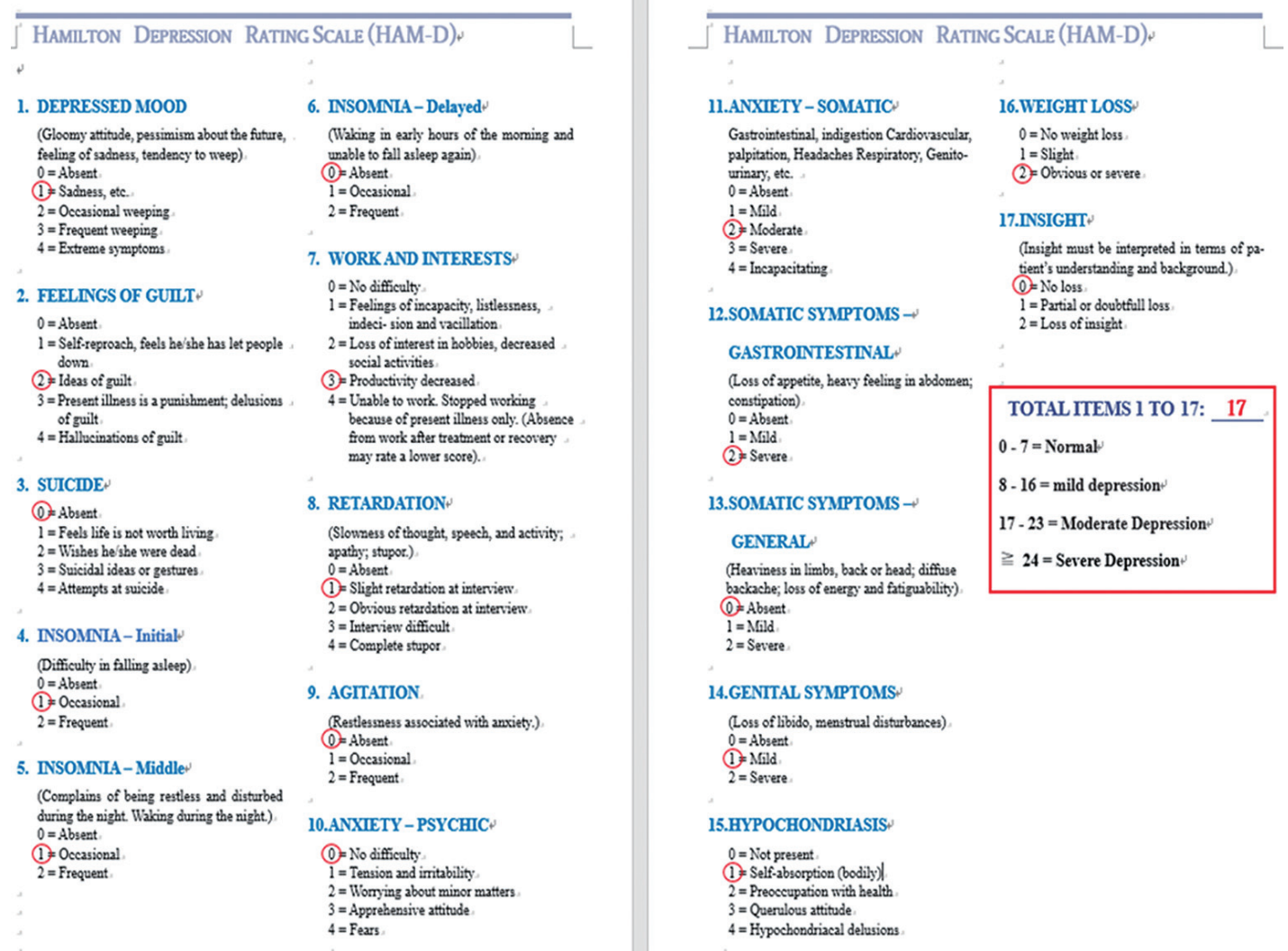

Figure 1. The HAM-D is designed to rate the severity of depression in patients. Although it contains 21 areas, it calculates the patient's score on the first 17 answers. The following severity ranges for the HAM-D are: no depression $(0-7)$, mild depression (8 - 16), moderate depression (17 - 23), and severe depression ( $\geq 24)$ [6]. Her HAM-D score was 17 points on admission.

nosed as anorexia due to depression.

\section{Case Report}

An 84-year-old woman started to gradually lose appetite. About a month later, she consulted her local doctor and was noted to have only superficial gastritis after a series of tests, including blood examinations, endoscopy, and abdominal ultrasound. She was prescribed a lansoprazole $15 \mathrm{mg} /$ day and regularly followed up at the hospital. She gained her appetite temporarily, but again lost her appetite and stopped having meals altogether after about a week. Because of this, she was advised admission by her local doctor.

Past medical history included total hysterectomy due to myoma at the age of 50 years. She was the oldest female of seven siblings, but was adopted and raised by her aunt since birth, with very little interaction with her biological parents and siblings. After graduating high school at the age of 21 years, she married an office worker. Moreover, she obtained a degree and managed a barbershop until the age of 74 years. Currently, she lives in a household of three with her son and his wife.

She was advised by her local doctor and visited our hospital at first on September 2. On physical examination, she was alert and conscious and had no apparent cognitive dysfunction.
She was $150 \mathrm{~cm}$ tall, weighed $38 \mathrm{~kg}$, and had a body mass index of $16.9 \mathrm{~kg} / \mathrm{m}^{2}$. Blood pressure was $102 / 64 \mathrm{~mm} \mathrm{Hg}$, pulse rate was 60 beats per minute, body temperature was $36.5^{\circ} \mathrm{C}$, and oxygen saturation was $96 \%$. The breasts and stomach area were unremarkable. Her hips were slightly bent and caused her to lean forward, but she could walk on her own. There was no anarthria or dysphagia. Mini-mental status examination score was $24 / 30$ points. Hamilton depression rating scale (HAM-D) [6] was $17 / 50$ points (Fig. 1). There was no obvious suicidal ideation. Laboratory findings showed no abnormalities. Thyroid hormone levels were also normal. Chest radiograph, electrocardiogram, and chest computed tomography (CT) showed no apparent abnormalities. Magnetic resonance imaging (MRI) of the head showed minimal diffuse white matter ischemic changes that were suggestive of atrophy in the frontal lobe, but this was expected of her age (Fig. 2). She was subsequently discharged and was advised to follow-up as an outpatient. On her outpatient visit, the patient mentioned that she did have any internal need or desire to eat. Although she was drinking $500 \mathrm{~mL}$ of milk every day, she lost $5 \mathrm{~kg}$ within the past month. Upon assessment, we noted that her physical strength was gradually getting weaker and that her ability to walk was declining due to disuse. As an outpatient, there was not enough time for the staff to talk and communicate fully with the patient. In addition, we judged that it was difficult to continue following her progress with tests as an outpatient and 

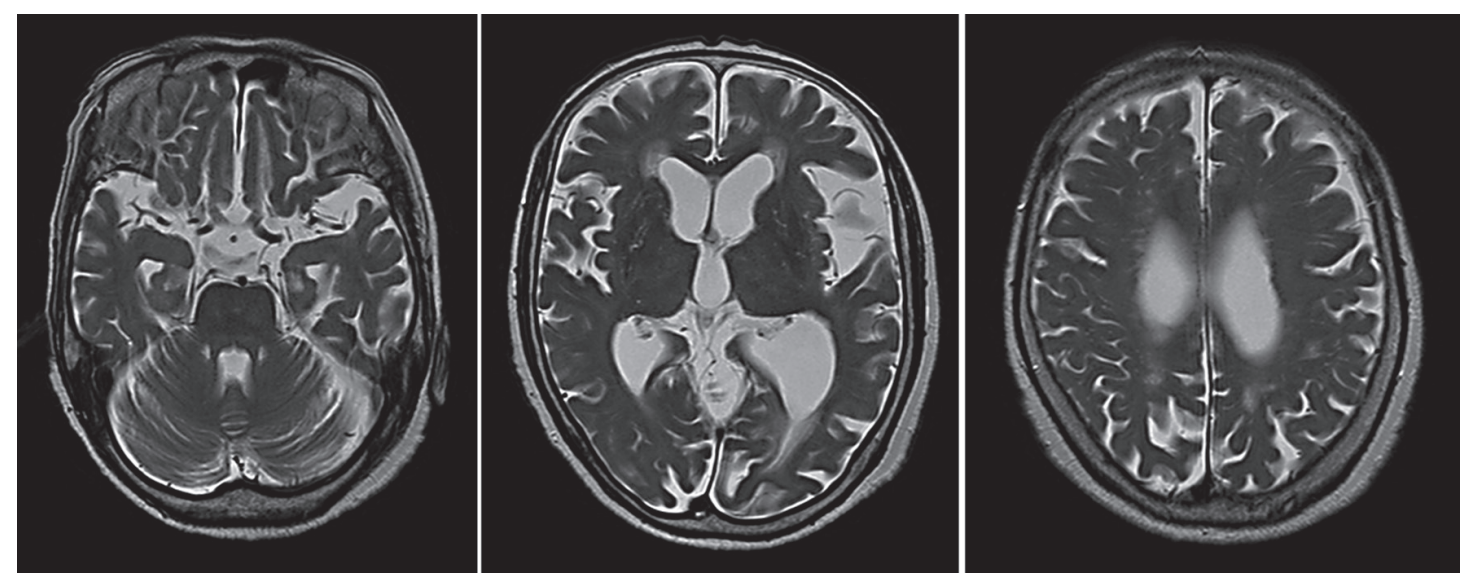

Figure 2. MRI of the head (T2-weighted sequences) showed some light scattering of ischemic changes in the white matter of the deep parts of the frontal lobe, but the atrophy is expected of someone her age.

admitted her to the hospital on September 26. Repeat upper gastrointestinal endoscopy and colonoscopy did not reveal any anatomic abnormalities. We continued to track her progress along with administration of vitamin tablets, fluid replacement, and physical rehabilitation to prevent muscle disuse.

During this admission, she started talking with us and we learned that the patient started to become emotionally unstable since the 2011 earthquake. She would get frightened of aftershocks during the night, became scared of being alone during the day, and would have sleepless nights because of persistent thoughts about the earthquake. She visited psychiatrists a few times and was prescribed sleep-inducing pills. About 2 years ago, she joined a senior club by a non-profit organization and started participating in activities, such as haiku, playing the harmonica, and using the computer, which were held four times a week. She recalled looking forward to each meeting until about 4 months before her hospitalization, when she was invited to attend a school reunion. Although she always looked forward to reunions, she did not think that it would be fun because only two of her best friends will be able to attend. She was unmotivated and decided not to attend. Later, at the senior club, she started feeling that her mind was not functioning as quickly as before and she grew impatient toward herself. Although her facial expressions were normal and conversations were smooth, it seemed as if she lacked the will to live and often blamed herself. After discussing with psychiatrists, we diagnosed her as having an eating disorder from geriatric depression.

We started administering the patient with $5 \mathrm{mg}$ of mianserin, $20 \mathrm{mg}$ of mirtazapine, and $25 \mathrm{mg}$ of dogmatyl. Subsequently, she was discharged from the hospital and was asked to continue to visit as an outpatient. About a month after discharge from the hospital, her appetite improved gradually, she started enjoying rehabilitation, and even started snacking. She no longer lacked the ability to enjoy experiences. About a month after she left the hospital, she came to the hospital on a silver car, was walking on her own, smiled often, and became her healthy self. At this time, she claimed that meals tasted good and was even eating a little too much. Thereafter, she continued to visit the hospital on a regular basis for checkups. About half a year after discharge from the hospital, her weight returned to $50 \mathrm{~kg}$.

\section{Discussion}

This was a case of an 84-year-old elderly woman who complained of loss of appetite and weight, but with no cognitive functional disorders and no apparent signs of emotional stress. The female presented in this report anorexia can be a symptom of depression and the treatment plan was devised accordingly. After multiple tests, it became apparent that she was suffering from an eating disorder. The biopsychosocial model has increased popularity not only in the medical and psychiatric fields but also in other various medical fields recently due to its consideration of multiple forms of etiological risks when determining how to most effectively understand and treat individuals for physical and mental illness. This model, in regard to depression, takes into account biological and psychological or cognitive, and social risk factors/contributors to depression when making diagnosis and treatment. The evidence regarding the cause of late life depression is addressed from a biopsychosocial perspective. Thorough understanding of the biopsychosocial model enabled diagnosis and appropriate treatment of early-onset geriatric depression. The individual-focalized and humanized approach, assisting the patient as singular being, was the best one achieving her biopsychosocial needs in this clinical practice. The successful outcome after a typical biopsychosocial model of psychotherapeutic intervention for eating disorders provided additional indirect evidence that she was indeed suffering from anorexia.

At an old age, there are various experiences of loss, including loss of health, social roles, and personal relationships. By listening carefully in this case study, it was apparent that the patient experienced much loss. Moreover, in geriatric depression, patients have few negative emotions, such as feeling depressed, sad, or any other subjective drops in mood; this implies that elderly individuals might find it difficult to explain their symptoms to others, and that the people around them might hardly notice any symptoms of depression. Moreover, patients with this condition might experience several physical malfunctions, such as 
Table 1. Factors That Influence Refusal to Eat in the Elderly

\begin{tabular}{l}
\hline Depression \\
Dementia \\
Anorexia nervosa \\
Organic disease (e.g., various cancers and endocrine disorders) \\
Alcohol dependence \\
Neurodegenerative diseases (e.g., Parkinson's disease) \\
Stroke sequelae \\
Other mental disorders \\
Unstable mental condition \\
Side effects of drugs \\
Senility \\
\hline
\end{tabular}

pain, numbness, loss of appetite, etc. At an old age, many people experience loss of spouses, friends, status, health, and financial assets, in addition to biological changes in the brain and body. In other words, from the biopsychosocial perspective, elderly individuals might easily become depressed. Moreover, looking at the suicide rates by age group, older people have a higher tendency to commit suicide than people in the other age groups. In elderly individuals with depression, complaints about a depressed mood are often few, and the symptoms are atypical. Compared with young people, older people have few symptoms that are characteristic of intrinsic depression, such as desperate ideation, decreased sexual desire, guilt feeling, and depressed mood. Physical discomfort and anxiety symptoms, such as hypochondria, general physical symptoms, impatience, and digestive system symptoms have been reported [7]. Elderly people with depression have less frequent symptoms of depressed mood, guilt feelings, and diligent thought, which are considered to be common in intrinsic depression; instead, excessive anxiety to physical symptoms and feeling of frustration are frequent.

This is thought to be one of the causes of the actual increase in the number of diseases with aging. Some common co-morbid conditions associated with depression are coronary artery disease in about $10-20 \%$ and endocrine disorders in $20-60 \%$. Additionally, the prevalence rate of anorexia is approximately $66 \%$ in patients with advanced cancer [8]. Furthermore, the mortality rate of depression has been reported to be high; this was true even in central nervous system diseases, such as dementia in about $10 \%$, Parkinson's disease in about $30 \%$, and stroke in $20-30 \%$ [9]. In the overwhelming majority of cases that appeared in literature, disordered patterns of eating, weight, and body image in the elderly were accompanied by co-morbid conditions of anxiety, depression, grief, or dementia $[10,11]$. There have been cases of presumed anorexia that have actually been due to hypothalamic tumors and dysfunction [12], but there was no evidence of any mass on CT or MRI. MRI is usually used to rule out organic causes for psychiatric symptoms. Specially, it is usefulness for diagnosis by exclusion of vascular depression or vascular dementia [13]. Therefore, MRI was very useful in our case (Fig. 2). In addition, anorexia and refusal to eat disorders in the elderly have several differential diseases (Table 1). There is also a need to carefully exclude these diseases and situations.

About $10 \%$ of the elderly who receive primary medical care have been diagnosed with depression. Untreated geriatric depression could heighten the risk of cognitive function disorders, physical complications, and death; therefore, it is extremely important to test and diagnose this condition. Anorexia has proven to be an independent predictor of mortality in elderly people [14]. Therefore, it is important to note that depression is a treatable disease even in the elderly and that patients require appropriate social support, as well as medical and mental therapies. In addition, depression in the elderly is an influential risk factor for suicide; in this sense, differences in depressive symptoms between young people and older people should be known and not overlooked.

In conclusion, this case report highlighted that in atypical populations, such as the elderly, there is a need to be aware of the possibility of eating disorders to decrease risks to health and even life due to a delay in the diagnosis. People suffering from geriatric depression often do not volunteer feelings of sadness, depression, or other negative emotions. The elderly have a tendency to get depressed due to biopsychosocial causes. More than $90 \%$ of patients with depression are thought to visit primary care physicians and the departments such as general medicine first instead of psychiatrists. Therefore, the effectiveness of primary care will become more important in the future and primary care physicians need to be vigilant for signs of depressive disorder to prevent suicide. We think that general medicine will have an more important part in medical practice in the future in Japan.

\section{Acknowledgments}

The authors especially thank the doctors of the Department of Psychiatry, Hospital of International University of Health and Welfare for their clinical advices and supports.

\section{Consent}

Written informed consent was obtained from the patient for publication of this case report and any accompanying images.

\section{Grant Support}

None.

\section{Competing Interests}

The authors declare that they have no competing interests.

\section{References}

1. Fornari V, Kent J, Kabo L, Goodman B. Anorexia nervosa: 
"thirty something". J Subst Abuse Treat. 1994;11(1):4554.

2. Marcus EL, Berry EM. Refusal to eat in the elderly. Nutr Rev. 1998;56(6):163-171.

3. Giannini AJ. Drug abuse and depression: possible models for geriatric anorexia. Neurobiol Aging. 1988;9(1):26-27.

4. Morley JE, Silver AJ, Miller DK, Rubenstein LZ. The anorexia of the elderly. Ann N Y Acad Sci. 1989;575:5058; discussion 58-59.

5. Russell J, Gilbert M. Is tardive anorexia a discrete diagnostic entity? Aust N Z J Psychiatry. 1992;26(3):429-435.

6. Zimmerman M, Martinez JH, Young D, Chelminski I, Dalrymple K. Severity classification on the Hamilton Depression Rating Scale. J Affect Disord. 2013;150(2):384388.

7. Hegeman JM, Kok RM, van der Mast RC, Giltay EJ. Phenomenology of depression in older compared with younger adults: meta-analysis. Br J Psychiatry. 2012;200(4):275-281.

8. Komurcu S, Nelson KA, Walsh D, Donnelly SM, Homsi
J, Abdullah O. Common symptoms in advanced cancer. Semin Oncol. 2000;27(1):24-33.

9. Robinson RG, Price TR. Post-stroke depressive disorders: a follow-up study of 103 patients. Stroke. 1982;13(5):635641.

10. Bernstein IC. Anorexia nervosa, 94-year-old woman treated with electroshock. Minn Med. 1972;55(6):552553.

11. Hall P, Driscoll R. Anorexia in the elderly - an annotation. Int J Eat Disord. 1993;14(4):497-499.

12. Plata-Salaman CR. Cytokines and anorexia: a brief overview. Semin Oncol. 1998;25(1 Suppl 1):64-72.

13. Aizenstein HJ, Baskys A, Boldrini M, Butters MA, Diniz BS, Jaiswal MK, Jellinger KA, et al. Vascular depression consensus report - a critical update. BMC Med. 2016;14(1):161

14. Landi F, Liperoti R, Lattanzio F, Russo A, Tosato M, Barillaro C, Bernabei R, et al. Effects of anorexia on mortality among older adults receiving home care: an observation study. J Nutr Health Aging. 2012;16(1):79-83. 